\title{
Unmanned Navigation of the 1/10 Vehicle Using U-SAT
}

\author{
Su Yong Kim ${ }^{1}$ and SooHong Park ${ }^{2}$ \\ ${ }^{1}$ Graduate student, Department of Mechanical Engineering, Pusan National University, \\ KumJung Ku, Pusan 609-735, Korea \\ syz2ang@hanmail.net \\ ${ }^{2}$ Department of Mechatronics Engineering, Dongseo University, \\ Sasang Ku, Pusan 617-716, Korea \\ shpark@dongseo.ac.kr
}

\begin{abstract}
In order for a vehicle to follow a predetermined trajectory accurately, its position must be estimated accurately and reliably. In this paper, we propose new lateral control methods for unmanned vehicles and a positioning system using ultrasonic waves. The positioning problem is considered as an important issue of control problem for unmanned navigation of a vehicle. Dead Reckoning is widely used for positioning of vehicle. However this method has problems because it accumulates estimation errors. We propose a new method to increase the accuracy of position estimation using the Ultrasonic Satellite system. It is shown that we will be able to estimate the position of vehicle precisely, in which errors are not accumulated. We also propose new lateral control methods including a new path planning method and a heading angle modulator. The experimental results show that the proposed methods enable accurate vehicle trajectory tracking under various environmental factors.
\end{abstract}

Keywords: Ultrasonic satellite system (U-SAT), unmanned navigation, lateral control.

\section{Introduction}

In modern society, the road capacity is limited, but the number of vehicles is increasing continuously. Therefore, the smooth traffic of vehicles becomes difficult, which increases the threat to the safety of drivers. Under such circumstances, research is under way worldwide to develop unmanned navigation including ITS (Intelligent Transportation System), PATH (Partners for Advanced Transit and Highway) and AHS (Automated Highway System) [1], [2], [3].

The prerequisite for such unmanned navigation is the positioning of a vehicle. Only when the position of the vehicle is known, the vehicle could be drive associated with specific routes, which is generated using the general information needed for unmanned navigation. To this end, researchers have conducted various studies on INS (Inertia Navigation System), GPS (Global Positioning System), Vision System, and Ultrasonic System [4], [5]. In the case of INS, which uses an accelerometer or a gyroscope, there is no limitation regarding the recognition area. However, accumulative errors will occur with the passage of running hours, even though it is easy to construct a path planning. GPS guarantees precise positioning, but it created cost problems and limitations in recognizing environments. In the case of Vision 
System, its image processing speed is improving, owing to the development of microprocessors, but it has failed to completely overcome the challenge of recognizing environments.

This paper proposes an Ultrasonic Satellite system (U-SAT) for the unmanned navigation of vehicles accordingly. One of the difficulties associated with the study of unmanned navigation, is to conduct a test to verify the algorithm using an actual car. To resolve such a difficulty, this study conducted unmanned navigation experiments indoors using a 1/10 vehicle and a U-SAT. In addition, overcome the limitation of the existing lateral control, this study suggests a new lateral control algorithm, in which the performance of the algorithm has been verified and evaluated.

\section{Position Estimation Using Ultrasonic Waves}

As shown in Fig. 1, the basic formulation of positioning using ultrasonic waves is to use Expression (1) in conjunction with Expression (2), to measure the Time of Flight (T.O.F), the gap between the time of transmission of ultrasonic waves $\left(T_{t}\right)$ and the time receives of the waves $\left(T_{r}\right)$. It is necessary to measure the T.O.F. precisely for exact positioning and, to this end, this study used the period detecting method [6]. Using the $40 \mathrm{kHz}$ MA40BR/S ultrasonic sensor of Murata and the radio frequency (RF) transmitting-receiving module, the point of time of ultrasonic wave transmission

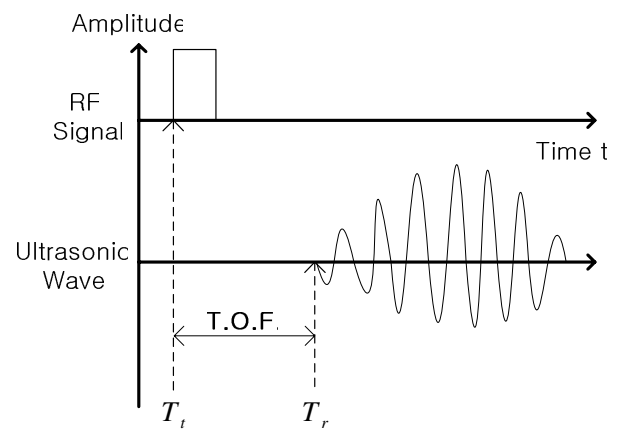

Fig. 1. Definition of T.O.F

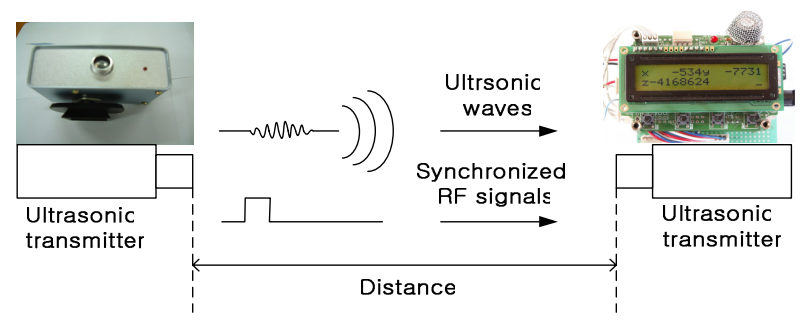

Fig. 2. Ultrasonic distance measurement system 
is measured. This study also constructed a ultrasonic distance measurement system as shown in Fig. 2 [7].

$$
\begin{gathered}
d=c \times \text { T.O.F } \\
c=331.5+0.60714 T
\end{gathered}
$$

Based on the ultrasonic distance measuring system shown in Fig. 2, this study constructed the U-SAT shown in Fig. 3. As shown in Fig. 3, the ultrasonic wave transmitter of Fig. 2 transmits ultrasonic waves and synchronized RF signals from four fixed positions. The ultrasonic receiver of Fig. 2 is attached on the moving object to receive ultrasonic waves and synchronized RF signals, as well as measuring the distances between ultrasonic transmitters and receivers. Based on this, the precise three-dimensional positions can be estimated through trilateration. In addition, ultrasonic waves are transmitted consecutively at the interval of 0.1 second in order to prevent interference of ultrasonic waves generated among each of the ultrasonic transmitters [8].

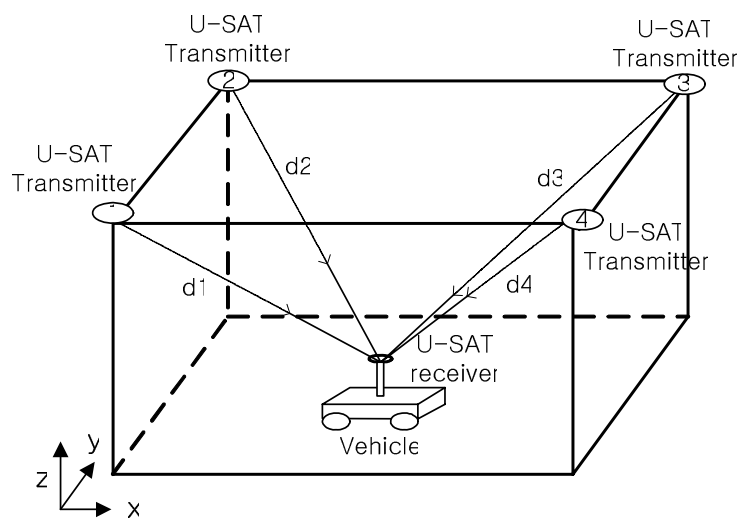

Fig. 3. Configuration of U-SAT

\section{Configuration and Modeling of the 1/10 Vehicle}

Fig. 4 indicates the configuration of a vehicle analogously reduced to $1 / 10$ of an actual vehicle. As shown in Fig. 4, the configuration of the 1/10 vehicle acquires the absolute position $(x, y)$ with the sampling time of 0.4 seconds through the U-SAT and obtains the heading angle $(\theta)$ from the electronic compass (CMP303-Robot Compass Module) at the interval of 0.1 second. The electronic compass has an error of about. The central processing part conducts wireless communications with the computer through a Bluetooth and receives data $(x, y, \theta, v)$ transmission and control inputs $(\delta, V)$ at the speed of $115200 \mathrm{bps}$. The steering part of the vehicle drives the HS 500 thermo motor of Hitec. The maximum right and left steering angle is $\pm 30^{\circ}$. 


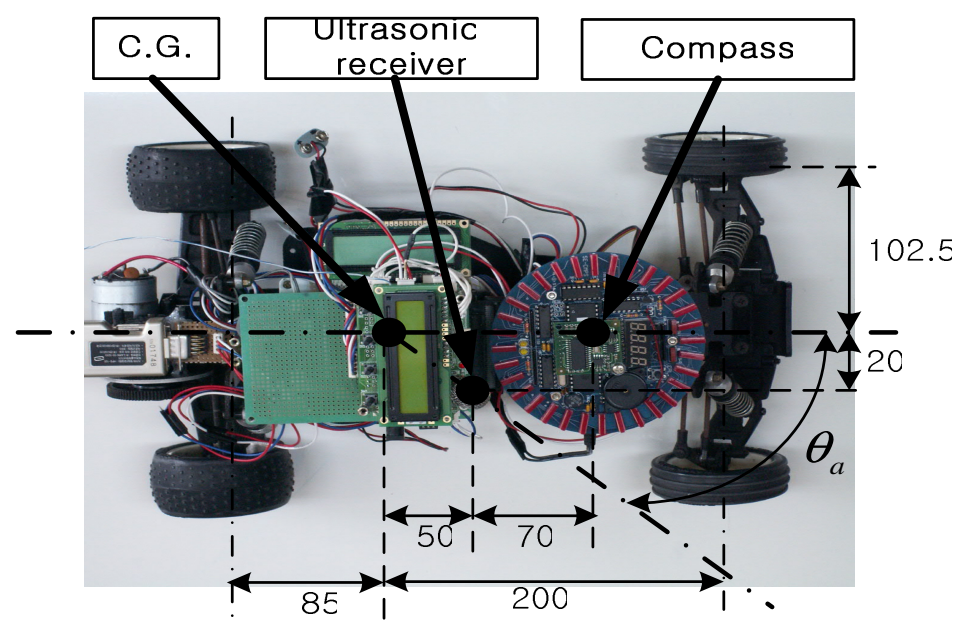

Fig. 4. Configuration of $1 / 10$ vehicle (uint: $\mathrm{mm}$ )

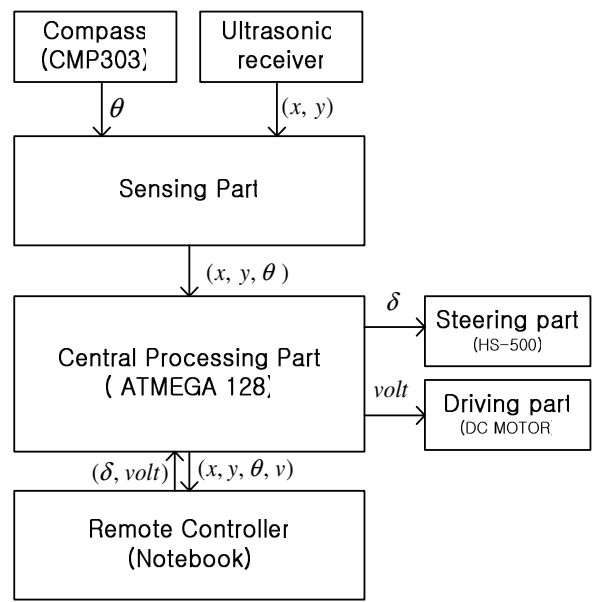

Fig. 5. Configuration of hardware

This study uses modeling that considers the look-down and actuator elements based on a bicycle model [4]. The bicycle model is a model that equalized two front wheels and two rear wheels as virtual wheels on the vehicle central line, respectively. This is to control steering and therefore, it ignores the vehicle roll and pitch movement. The premise is that the difference of angles in the small yaw rate and the longitudinal speed are maintained constantly. Linearization takes place under these conditions. 
The dynamic equation is expressed in the form of the differential equation like Eq. 3 . The parameters are shown in Table 1.

$$
\begin{gathered}
{\left[\begin{array}{c}
\dot{v} \\
\dot{r} \\
\dot{\theta}
\end{array}\right]=A\left[\begin{array}{c}
v \\
r \\
\theta
\end{array}\right]+B \delta_{f}} \\
A=\left[\begin{array}{ccc}
-2\left(C_{f}+C_{r}\right) / m u & -u-l\left(a C_{f}-b C_{r}\right) / m u & 0 \\
-2\left(a C_{f}-b C_{r}\right) / I_{z z} & -2\left(a^{2} C_{f}+b^{2} C_{r}\right) / I_{z z} u & 0 \\
0 & 1 & 0
\end{array}\right] \\
B=\left[\begin{array}{c}
2 C_{f} / m \\
2 a C_{f} / I_{z z} \\
0
\end{array}\right], C=\left[\begin{array}{lll}
0 & 0 & 1
\end{array}\right], D=0
\end{gathered}
$$

Where,

$$
\begin{array}{ll}
a & : \text { Distance from the vehicle C.G. to front axle } \\
b & : \text { Distance from the vehicle C.G to rear axle } \\
C_{f} / C_{r}: & \text { front/rear tire cornering stiffness [N/rad] } \\
I_{z z} & : \text { yaw moment of inertia } \\
l & : \text { wheel base l= a+b } \\
m & : \text { vehicle total mass } \\
r & : \text { yaw rate } \\
u & : \text { longitudinal velocity of the vehicle at C.G. } \\
v & : \text { side-slip velocity of the vehicle at C.G. } \\
\delta_{f} & : \text { front steering angle input }
\end{array}
$$

Table 1. Parameters of $1 / 10$ car

\begin{tabular}{|l|lc|}
\hline Parameter & \multicolumn{2}{|c|}{ value } \\
\hline$m$ & 2.105 & {$[\mathrm{~kg}]$} \\
\hline$l$ & 285 & {$[\mathrm{~mm}]$} \\
\hline$a$ & 200 & {$[\mathrm{~mm}]$} \\
\hline$b$ & 85 & {$[\mathrm{~mm}]$} \\
\hline$C_{f}$ & 0.191 & {$[\mathrm{~N} / \mathrm{rad}]$} \\
\hline$C_{r}$ & 0.51 & {$[\mathrm{~N} / \mathrm{rad}]$} \\
\hline
\end{tabular}

\section{Path Planning Method and Unmanned Navigation Algorithm}

As indicated in Fig. 6, a new path planning method is generated in a point-to-point manner. First of all, the vehicle moves toward the target route point $P_{p}(i)$. When the 


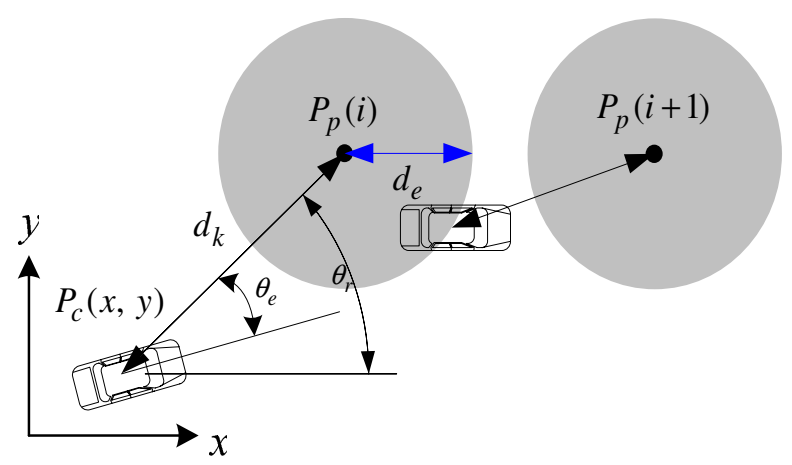

Fig. 6. Path planning, method and Lateral control algorithm

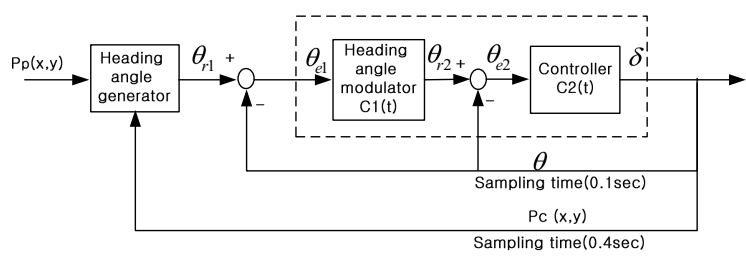

Fig. 7. Block diagram of feedback loop by using heading angle modulator

vehicle's center of gravity comes into a certain radius $d_{e}$ of the target route point $P_{p}(i)$, the vehicle is considered to have reached the target route point. Then, the target point is converted to the next target route point $P_{p}(i+1)$ iteratively. Here, the purpose of the radius $d_{e}$ is to facilitate natural movement to the next target route point. In this study, the vehicle speed is set at $40 \mathrm{~cm} / \mathrm{s}$ and $d_{e}$ at $25 \mathrm{~cm}$ in consideration of position errors.

Next, the lateral control algorithm controls the heading angle of the vehicle so that the error $\theta_{e}$ between the target route point $P_{p}(i)$ and the heading angle can be maintained at zero, as shown in Fig 6. As for the feedback loop, when the target route point $P_{p}(i)$ is given as shown in Fig. 7, the vehicle position $P_{c}(x, y)$ at that time is feedbacked to generate the target angle $\left(\theta_{r 1}\right)$ of the vehicle. This angle is set as a basic input and the heading angle error $\left(\theta_{e 1}\right)$ is made to converge at zero using the heading angle $(\theta)$ which comes in through the electronic compass. Then, the vehicle moves to the target route point $P_{p}$. The heading angle sampling time on the electronic compass is four times faster than the positioning sampling time of the U-SAT. Therefore, when the compensation is made simply by feedbacking the heading angle, $\theta_{e 1}=\theta_{r 1}-\theta$ is sent to zero. However, the vehicle can't realistically approach the target route point $P_{p}$. To overcome this problem, this study proposes a new lateral controller using the heading angle modulator $C_{1}(t)$, shown in Fig. 7. $C_{1}(t)$, as shown 
in Fig. 7 can be expressed as Eq. 4. Here, $d_{k}$ represents the distance error between the current vehicle positions while $P_{c}$ and $K_{r}$ indicate the heading angle modulation gain. $K_{r}$, which is determined by the rotational radius of the vehicle and the distance to $P_{p}$. Based on this, unmanned navigation of the $1 / 10$ vehicle is conducted through lateral control. Also, the performance of the proposed lateral control algorithm is verified.

$$
\theta_{r 2}(t)=\theta_{r 1}(t)+K_{r} \frac{\theta_{e 1}(t)}{d_{k}(t)}
$$

\section{Unmanned Navigation Experiment}

The lateral control experiment of the $1 / 10$ vehicle is conducted in an indoor space with an area of $7000 \mathrm{~mm} \times 4000 \mathrm{~mm}$. Fig. 8 indicates the target route points $P_{p}(i)(i=1, \cdots, 20)$ of the vehicle. The points are located at an interval of $50 \mathrm{~cm}$ and the range of the target route points were set at $25 \mathrm{~cm}$. In the unmanned navigation experiment, the first vehicle starts from the position of " 5000,3500 ". The first target route point is set at the position of " 2500 , 3000". While changing its target route points, the vehicle continues to move counterclockwise along the track at a constant speed of $400 \mathrm{~mm} / \mathrm{s}$.

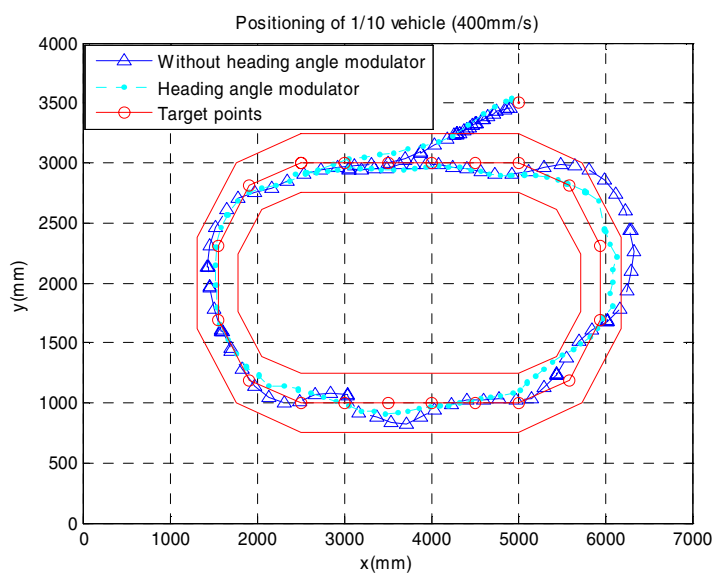

Fig. 8. Experiment result of unmanned navigation

Fig. 8 shows the result of an unmanned navigation experiment using the existing PID controller. They indicate that the vehicle travels in a relatively stable manner along the target points, but it deviates varies the route from time to time. To overcome such a problem, a lateral controller equipped with a heading angle modulator is employed. The experiment result is shown in Fig. 8. The experiment include the heading angle modulator, the vehicle follows the track well within an error range of 


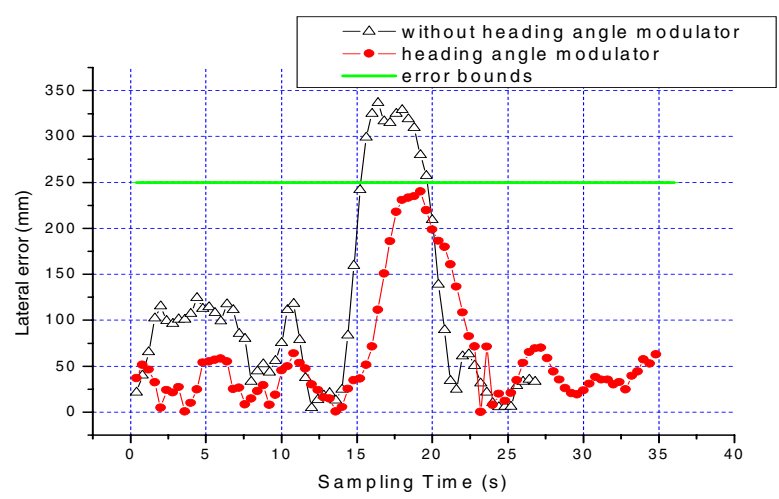

Fig. 9. Experiment result of lateral errors

$250 \mathrm{~mm}$, which is the range of the target route points. The lateral errors of the experiments are shown in Fig. 24. In the case using the existing PID controller, the maximum error is $336 \mathrm{~mm}$ and the average error is $109 \mathrm{~mm}$. The case include the heading angle modulator, however, the maximum error fell to $240 \mathrm{~mm}$ and the average error to $61 \mathrm{~mm}$. The latter represents much more stable performances than the former. Its driving performance can be said to be stable since the maximum error range is below $250 \mathrm{~mm}$. Through the unmanned navigation experiment, this study can confirm that unmanned navigation was (is) possible with the application of the U-SAT and that the performance of the lateral control algorithm added with a heading angle modulator was better than that of the (the) existing method. In addition, the algorithm verification was conducted easily with the use of the $1 / 10$ vehicle.

\section{Conclusion}

While overcoming the shortcomings of the existing positioning methods, this study suggests an U-SAT which enables precise positioning indoors, in addition to the configuration of the system. Based on this, the study used the U-SAT which is needed for the unmanned navigation of vehicles. In addition, this study has developed an unmanned navigation system for a $1 / 10$ vehicle and propose a new lateral control algorithm which is suitable for the system. Also, we have applied the algorithm and evaluated the performances. Based on the experiment results, this study has verified the appropriateness of the proposed lateral control algorithm, making it possible to conduct more stable unmanned navigation. In addition, the 1/10 vehicle developed with the unmanned navigation system has removed the risks that can be found in actual vehicle experiments. As this vehicle enables various tests in an easier and more convenient way, it could be used as a pre-test vehicle applicable before actual car tests. With respect to the research challenges to be addressed in the future, the USAT shows rather large errors for high-speed vehicle driving and therefore, further research will be necessary on this matter. 


\section{References}

1. Lappe, M., Raushecker, J.P.: A Neural Network for the Processing of Optic Flow from EgoMotion and Higher Mammals. Neural Computation 5(3), 374-391 (1993)

2. Kim, S., Kim, J.: Research of the Unmanned Vehicle Control and Modeling for Obstacle Detection and Avoidance. Transactions of KSAE, 11(5), 183-192 (2003). van Leeuwen, J. (ed.): Computer Science Today. Recent Trends and Developments. Lecture Notes in Computer Science, vol. 1000. Springer, Heidelberg (1995)

3. Ackermann, J., Guldner, J., Utkin, V.I.: A robust Nonlinear Control Approach to Automatic Path tharcking of a car. In: IEEE control conference, (389), pp. 196-201. IEEE, Los Alamitos (1994)

4. Lappe Tanagiya, M., Tomita, M., Tsugawa, S.: Effects of Field of View on Lateral Control Performance in a Vision_Based Autonomous Vehicle. JSAE Review 20, 499-504 (1999)

5. Abbott, E., Powell, D.: Land-Vehicle Navigation Using GPS. Proceedings of the IEEE 87(1) (January 1999)

6. Lee, D.H., An, H.T., Baek, K.R., Lee, M.H.: A study on resolution Enhancement in the detection of ultrasonic signal. In: The KSAE Annual Fall Conference, Branch of Busan Ulsan Gyeongnam, pp. 95-102 (2003)

7. Arai, T., Nakano, E.: Development of Measure Equipment for Location and Direction(MELODI) using Ultrasonic Waves. Transactions of the ASME 105, 152-156 (1983)

8. Kim, S.Y., Lee, D.H., An, H.T., Lee, S.H., Koh, Y.H., Lee, M.H.: Analysis of UPS (ultrasonic positioning system) using DGPS. In: IECON 2004. 30th Annual Conference of IEEE, vol. 1, pp. 609-613. Industrial Electronics Society (2004) 